\title{
Reconfiguring the radiology leadership team for crisis management during the COVID-19 pandemic in a large tertiary hospital in Singapore
}

\author{
Kiang Hiong Tay ${ }^{1,2} \cdot$ Chin Chin Ooi ${ }^{2,3} \cdot$ Muhammad Illyyas Bin Mahmood ${ }^{3} \cdot$ Lian Ping Aw ${ }^{3} \cdot$ Lai Peng Chan $^{2,4}$. \\ David Chee Eng Ng${ }^{2,5} \cdot$ Bien Soo Tan ${ }^{1,2}$
}

Received: 19 May 2020 / Revised: 7 July 2020 / Accepted: 29 July 2020 / Published online: 11 August 2020

(C) European Society of Radiology 2020

\begin{abstract}
The coronavirus 2019 (COVID-19) outbreak poses a serious public health risk. To date, the disease has affected almost all countries in the world. The enormous scale of the outbreak and the relative lack of knowledge and information regarding a new virus, as well as the unpredictability of events, make it challenging for leadership teams to respond. This paper shares how we have reconfigured our radiology leadership team into a smaller disease outbreak task force (DOTF) to respond and coordinate all related efforts during this ongoing COVID-19 pandemic. The DOTF format is modelled after the military with domain groups looking at manpower, intelligence, operations, and logistics matters on a daily basis so that timely decisions can be made and action plans executed promptly. In managing the DOTF, discipline, flexibility, and teamwork are key principles, and these are built upon a strong foundation of focus on infection prevention and control, and patient and staff safety as well as staff well-being. The DOTF has positioned us well to confront the many challenges to date. We believe it will also help us navigate the complex issues that will arise with future surges in cases and in formulating strategies to manage exit from the present and future lockdowns.

\section{Key Points}

- In a pandemic, regular and directed meetings by a smaller leadership core group are required, for prompt decision making and execution of action plans.

- The military format, with domain groups to look at manpower, intelligence, operations, and logistics matters, is useful in managing a pandemic.

- Discipline, flexibility, and teamwork with strong focus on infection prevention and control, and patient and staff safety as well as staff well-being are key principles for leadership teams managing a pandemic.
\end{abstract}

Keywords COVID-19 · Coronavirus · Disease outbreaks · Radiology · Leadership · Pandemics

\section{Abbreviations and acronyms}

COVID-19 Coronavirus disease 2019

CT Computed tomography

DORSCON Disease Outbreak Response

System CONdition
DOTF Disease outbreak task Force

IPC Infection prevention and control

MRI Magnetic resonance imaging

PPE Personal protective equipment
Kiang Hiong Tay

tay.kiang.hiong@singhealth.com.sg

1 Department of Vascular and Interventional Radiology, Division of Radiological Sciences, Singapore General Hospital, Block 2 level 1, Outram Road, Singapore 169608, Singapore

2 Duke-NUS Medical School, 8 College Road, Singapore 169857, Singapore
3 Radiography Unit, Division of Radiological Sciences, Singapore General Hospital, Outram Road, Singapore 169608, Singapore

4 Department of Nuclear Medicine and Molecular Imaging, Division of Radiological Sciences, Singapore General Hospital, Outram Road, Singapore 169608, Singapore

5 Department of Diagnostic Radiology, Division of Radiological Sciences, Singapore General Hospital, Outram Road, Singapore 169608, Singapore 
PRD Preparedness and response department

SARS Severe acute respiratory syndrome

\section{Introduction}

During an infectious disease epidemic, timely decisions need to be made and plans executed promptly to prevent widespread transmission. In the early phase of a disease outbreak, information tends to be incomplete and decisions have to be made based on fundamental infection prevention and control (IPC) principles as well as experiences from past disease outbreaks. As an epidemic unfolds, there will be need for leadership with foresight to anticipate potential issues, to manage them, or at least to prevent them from escalating [1].

Radiology services are a critical cog in an institution's response to a disease outbreak as imaging is a key component in the detection of infected patients and radiology facilities are potentially a nidus for intrahospital disease transmission $[2,3]$. The existing organizational structures of radiology departments are designed to cater to day to day operations as well as to facilitate mid- to long-term strategic planning. In large departments, depending on how the leadership structure is configured, the day to day operations are largely delegated to modality imaging teams and subspecialty radiology teams, often with significant autonomy. Mid- and longer-term decisions are made by a larger management team, usually through consensus and typically after comprehensive discussions and assessments. Such an organizational structure may be able to manage a minor crisis but is less likely suited for handling disruptive and dynamic major events like pandemics. In these situations, there is little room and time for lengthy discussions and prompt decision making, often based on limited information, is critical. We share how we have reconfigured our radiology leadership team into a disease outbreak task force (DOTF) to respond and coordinate all related efforts during this ongoing coronavirus disease 2019 (COVID-19) pandemic.

\section{Lessons from SARS}

Singapore was badly affected by the severe acute respiratory syndrome (SARS) epidemic in 2003. There were 8099 cases worldwide and Singapore had the third highest number of cases $(n=238)$ in the world. The case fatality rate was $13.8 \%$ in Singapore, compared with the worldwide case fatality rate of $9.6 \%$. There were extensive intrahospital transmissions, with cross infection among healthcare workers, inpatients, outpatients, and visitors. In fact, $40.8 \%$ of the SARS patients in Singapore were healthcare workers, of whom five of them (two doctors, two nurses, and one healthcare attendant) died [4].

Following SARS, our country has been systematically taking steps to improve our capability to deal with another major disease outbreak. The Singapore government introduced the DORSCON (Disease Outbreak Response System CONdition) framework in 2006 to guide response in an infectious disease outbreak situation [5]. This framework is not only designed for healthcare, but it also enables a "whole-of-government" response with formal platforms for inter-ministerial and cross-agency coordination, providing strategic and political guidance on a range of related issues such as border control, economic sustainability, security, transport, and education. Within the Ministry of Health (MOH), a disease outbreak team is responsible for medical response, surveillance, isolation, contact tracing, quarantine, and provision of medical care. Similar disease outbreak teams are established in all major public healthcare institutions to interface with $\mathrm{MOH}$ and coordinate responses and efforts in their respective institutions. There is also a communications component, which conveys the health impact of the disease to the general population and to advise them how to respond [6].

Our institution has a Preparedness and Response Department (PRD) to oversee civil emergencies, disease outbreaks, and business continuity planning. This department has representation from all relevant medical, nursing, allied health, and operations departments within the institution. Meetings and table-top exercises are conducted regularly and pertinent policies and standard operating procedures are formulated and updated. As major infectious disease outbreaks are uncommon, most of the exercises have focused on mass casualty response and business continuity.

\section{Existing radiology leadership organization structure}

At our institution, a 1773-bed tertiary hospital, the departments of Diagnostic Radiology, Vascular and Interventional Radiology, and Nuclear Medicine \& Molecular Imaging are organized under a division of Radiological Sciences. The faculty of the three departments together with radiographers, imaging technologists, nurses, and related support staff bring the total manpower strength of the division to over 700 . The division management committee, responsible for the day to day operations, is made up of key appointment holders of the departments and imaging modalities (General Radiography, CT, MRI, Ultrasound, Mammography, Interventional Radiology, and Nuclear Medicine) as well as leaders for nursing, radiography, support staff, administration, logistics, 
quality management, imaging informatics, and service development. This management committee has a total of $31 \mathrm{mem}-$ bers and meets fortnightly to coordinate and deliberate on major decisions, while allowing the various units to operate autonomously. The division also nominates representatives to participate in our institution's PRD meetings.

\section{Response to the COVID-19 outbreak}

The first confirmed COVID-19 case in Singapore was diagnosed in our institution on 23 January 2020. Our radiology management committee had convened daily to start preparations for the anticipated disease outbreak. We soon realized our existing management committee was too unwieldy for quick decision making and many of the committee members were not able to attend the increased frequency of meetings.

A smaller leadership group was quickly reconfigured into a DOTF to handle all matters related to the epidemic. The DOTF comprised the division senior management namely the division chair, assistant division chair, heads of the three clinical departments, leaders of radiography, nursing, clinical operations, imaging informatics, and administration. The general radiography manager was incorporated into our DOTF as general radiography became the key imaging modality for COVID-19 in our practice, as was our logistics manager as we felt it was critical to track of our personal protective equipment (PPE) stocks and usage. We also incorporated personnel with experience in fast-paced operations (during their stint in military service) even though they did not hold current positions in our management committee. With this, we reduced our number to a more manageable 17 members for the DOTF.

One additional feature of our DOTF was that several key leaders had held significant leadership roles during SARS. This proved to be valuable during the initial outbreak period as much of the initial response could then be implemented based on our experience with SARS [2, 7]. However, as the outbreak evolved into a pandemic, it became clear that COVID-19 had some different features and new approaches had to be formulated [8-10].

Our division chair is an integral member of our institutional DOTF. During the onset of the outbreak, our institutional DOTF met daily in the morning and disseminated information and instructions to the rest of the hospital through a set of routine instructions in the afternoon. We decided that our DOTF would meet daily in the late afternoon so that information from the institution can be incorporated into our decisionmaking process and action plans. The intent was that the meeting should not last longer than an hour.

Additionally, we felt that it was important that a smaller core leadership group meets first thing in the morning daily to review overnight events and changes, update the manpower situation, and anticipate issues for the day. This group comprised 10 members, and the morning meeting lasts about $20 \min [11]$.

\section{Radiology DOTF format and framework}

To cater for the rapidly changing scenarios and operational demands, we decided that our DOTF format and framework would be mirrored after the military format. In Singapore, we have a conscript armed forces, and all males would have experienced military training, and are familiar with this format. The female members of our staff, having fathers, husbands, partners, brothers, and sons conscripted, were also attuned to this military concept. Our DOTF was divided into domain groups looking at manpower, intelligence, operations, and logistics matters, corresponding to the key staff functions of a military organization [12-14]. This use of a military format has been advocated as a systematic and effective method for handling a crisis [15]. Briefly, the military staff structure and functions are divided into eight domains from $\mathrm{G} 1$ to $\mathrm{G} 6$ and $\mathrm{G} 8$ to $\mathrm{G} 9$ ( $\mathrm{G}$ stands for general staff and there is no G7) where $\mathrm{G} 1$ is responsible for personnel or manpower issues, $\mathrm{G} 2$ is in charge of intelligence gathering, $\mathrm{G} 3$ is responsible for operations, G4 looks after logistics, G5 is responsible for mid- to long-term planning, G6 is in charge of signal/ communications, G8 is responsible for financial management, and G9 deals with civilian affairs [13]. We adopted the first four domains for our DOTF as we felt they were the most relevant.

\section{Manpower}

The manpower domain tracks the number of staff at work and the deployable staff numbers. Staff on sick leave or on quarantine are monitored (Table 1). All our staff are required to submit body temperature readings twice daily and this domain monitors the readings and scans where staff are deployed to detect any potential fever clusters forming with our division. When physical distancing was implemented, this domain coordinated the roster of staff working from home. The manpower domain also formulated a division communications strategy, to ensure all decisions made reach the ground for consistent implementation. The wellbeing of all staff is coordinated with our division staff experience team, resulting in initiatives like regular distribution of individual care packs to boost staff morale.

\section{Intelligence}

The intelligence domain gathers all information relevant to COVID-19, including instructions and directives from our institution and $\mathrm{MOH}$. They also scan the medical literature and various media platforms for new scientific evidence or data that could impact our decisions or actions. One such example was the need for physical distancing to be implemented within our work environment, a measure that was 
Table 1 Manpower movement update during daily Disease Outbreak Task Force (DOTF) meeting

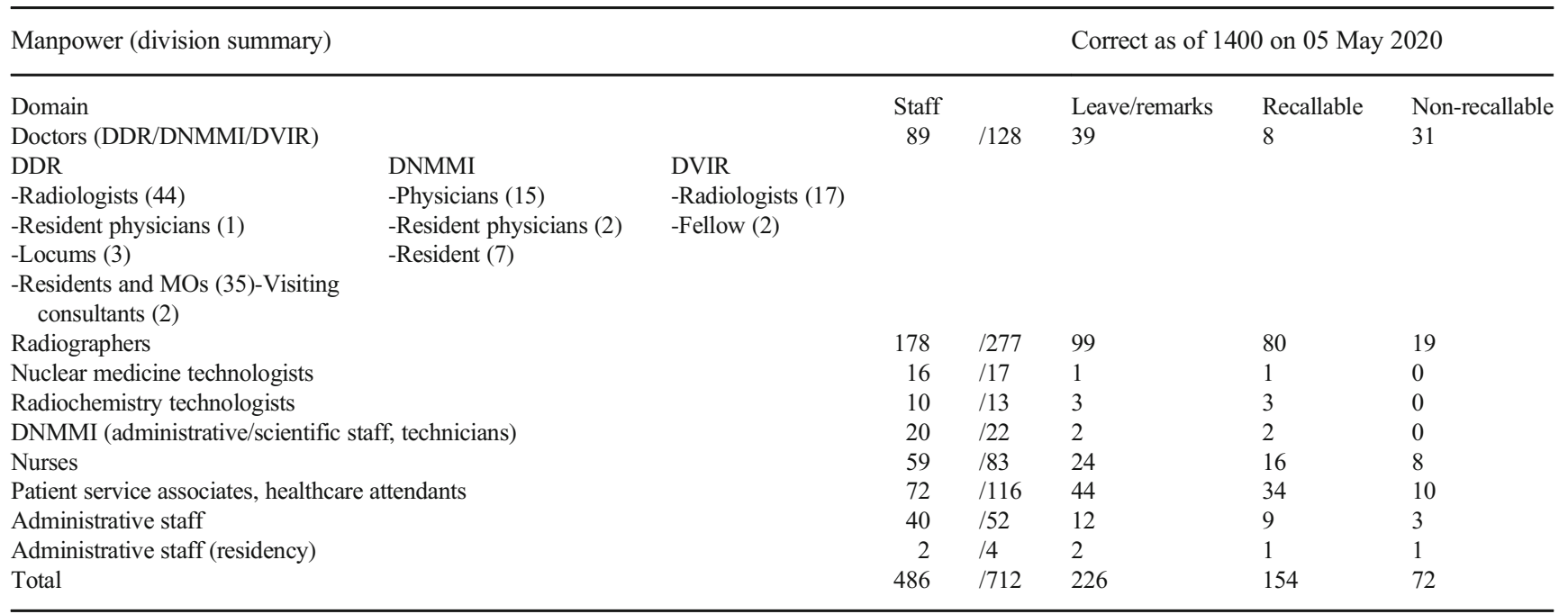

DDR, Department of Diagnostic Radiology; DNMMI, Department of Nuclear Medicine and Molecular Imaging; DVIR, Department of Vascular and Interventional Radiology

not as robustly imposed during SARS [16]. More recently, with the deluge of publications on COVID-19, a more organized and systematic review of the emerging literature became necessary.

\section{Operations}

The operations domain deals with formulating and implementing workflow changes and staff deployment related to handling of COVID-19 patients and patients suspected of or at risk of COVID-19. These include drawing up detailed IPC guidance for the various imaging settings and scenarios, in alignment with our hospital IPC team's recommendations [7, 17, 18]. Refresher training in proper use of PPE and powered air-purifying respirator was organized.

The domain also monitors workload data of the various imaging modalities closely to anticipate surges or decline in demand so that manpower and resources can be redeployed accordingly. In particular, the demand for portable radiography at various locations, namely the isolation wards, our higher risk acute respiratory infections wards, the emergency department, and fever screening areas are tracked daily. When a partial national lockdown was introduced, the need for readjusting imaging appointments was also initiated and worked on with all stakeholders. In collating this data, it is useful to have software (Tableau Software) that facilitates real-time analysis, so that short-term trends in terms of weeks and days can be identified and compared with historical data. Another strategy that was adopted was to insert a radiology faculty into our infectious disease team, allowing us real-time information and the ability to shape our imaging service response [19].

\section{Logistics}

The logistics domain keeps track of all consumables, imaging equipment, reporting workstations, and real estate management. Equipment under repair or servicing is monitored closely to ensure minimal downtime. Logistics also monitors PPE usage closely and ensures adequate stocks are at hand. This domain is responsible for mask distribution to minimize wastage. When it was clear that physical distancing was necessary, the reconfiguration of all our work areas and staff rest areas was under its purview.

\section{Meetings}

The operations domain is tasked to conduct these meetings and serve as the secretariat. Our DOTF meeting agenda is composed of a fixed format to ensure that no key issue is missed (Table 2). At the DOTF meetings, each domain will provide updates of their respective areas.

The agenda also includes reviews of problem areas that have been highlighted. One example is that a daily walkabout has been instituted to ensure IPC standards, address patient and staff safety issues, and identify ground issues. Key findings during these walkabouts are presented as part of the agenda. Feedback received from staff were collated and addressed, and where there were good suggestions from the ground, these were rapidly implemented.

Small teams are also assigned as necessary to look after specific projects that require fast turnaround, for example, planning the radiology support when our institution decided to convert a multi-storey car park on campus into a fever screening area at short notice. This multi-storey car park had been designed and pre-built to be easily reconfigured during a 
Table 2 Disease outbreak task force (DOTF) meeting agenda format

\begin{tabular}{|c|c|}
\hline & Agenda \\
\hline \multicolumn{2}{|c|}{ Morning meeting } \\
\hline 1. & $\begin{array}{l}\text { Overnight events } \\
\text { - Issues highlighted by on-call team } \\
\text { - Routine instructions, MOH (Ministry of Health) circulars/updates }\end{array}$ \\
\hline 2. & Major events for the day \\
\hline 3. & $\begin{array}{l}\text { Manpower } \\
\text { - Key appointment holder major movement } \\
\text { - Roster issues }\end{array}$ \\
\hline 4. & Operations issues and concerns \\
\hline 5. & $\begin{array}{l}\text { Logistics } \\
\text { - Status of imaging equipment, reporting workstations, and procedural rooms }\end{array}$ \\
\hline 6. & Management guidance and watch areas \\
\hline \multicolumn{2}{|c|}{ Evening meeting } \\
\hline 1. & Review of previous minutes and action items \\
\hline 2. & $\begin{array}{l}\text { Manpower status } \\
\text { - On duty, off duty, medical leave, other leave, secondment, deployable strength }\end{array}$ \\
\hline 3. & Intel (intelligence) updates \\
\hline 4. & $\begin{array}{l}\text { Logistics } \\
\text { - Status of imaging equipment, reporting workstations, and procedural rooms } \\
\text { - Usage rate and local stockpile of key consumables items }\end{array}$ \\
\hline 5. & Training \\
\hline 6. & $\begin{array}{l}\text { Safety } \\
\text { - Walkabout findings }\end{array}$ \\
\hline 7. & AOB (any other business) \\
\hline
\end{tabular}

disease outbreak, and housed a lead-lined room for radiography.

Other issues that are identified but do not fall under the direct purview of the subgroups are delegated to additional teams to address, for example, education and research matters. At the end of each DOTF meeting, information and instructions are collated and packaged for dissemination to the rest of the staff in the division. The DOTF format and framework are strictly adhered to and all discussions are minuted so that outstanding issues are addressed or followed up.

The DOTF meetings were conducted in person initially but as the outbreak progressed and physical distancing measures were introduced, the meetings are now conducted using video conferencing platforms (Zoom Video Communications and Cisco Webex). Over time, the frequency of the DOTF meetings has decreased to three times a week although the core leadership group continues to meet daily in the morning.

After the initial response period to the outbreak, we have also resumed our fortnightly management committee meetings to address ongoing business including the implementation of annual workplans and budgets, planned equipment procurement, and addressing how these may have to be adjusted in terms of timing in the light of the pandemic.

\section{Communications}

The best laid plans will not lead to robust implementation without a comprehensive communications strategy. To this end, we realized that to reach out to more than 700 staff effectively, multiple platforms had to be adopted. Official communications were sent out daily via email. For other relevant information including staff well-being initiatives, an approved social media platform was utilized (Workplace by Facebook). In addition, every team was informed to set up secure text messaging communications chat groups using the institutional approved platform (TigerConnect) for work-related matters including patient information so that we adhere to our privacy laws. Staff chat groups using popular social media platforms (WhatsApp Messenger) were also encouraged for non-workrelated communications, to allow staff to stay socially connected.

\section{Outcomes}

At the time of writing (30 June 2020), there has been 43,907 cases of COVID-19 reported in Singapore and our institutional has managed 1491 inpatients cases in total; 39 cases are 
currently admitted [20]. We have performed $1371 \mathrm{X}$-rays, five ultrasounds, 38 CT scans, and one MRI scan on COVID-19positive patients. With this leadership strategy, only one member of our division has been infected, from contact within the community, and we have had no incidence of in-hospital transmission among our staff.

Since the onset of the outbreak, major radiology service initiatives that we have had to undertake include the implementation of portable X-ray and ultrasound services in highrisk areas, setting up of a new institution fever screening area with X-ray facilities in a multi-storey carpark to complement our emergency department, and installation of a CT scanner (loaned to us without cost by an industry partner) to increase capacity for the duration of the pandemic. We have also managed a huge surge of cases nationally for the past 3 months.

One component of effective leadership is ensuring that we do not become complacent when we appear to be having the crisis under control, but to continue to think and to plan ahead strategically [21]. In addition to reacting to the fast-paced changes, our DOTF has also been tasked to look toward the future to anticipate the "new normal" of providing imaging and image-guided treatments [22]. As we serve more than one imaging location, we are investigating how we can better plan and interface with our patients so that the appropriate imaging study can be delivered at the right time and at the appropriate site while reducing patient dwell time in our facilities. We are also piloting teleconsultations to reduce the need for patients to travel to our facilities. Many of the communications platforms we have now embraced will be key to our future initiatives in management, the delivery of clinical service, and in education.

An interesting outcome that has also arisen from this crisis has been the opportunity to identify colleagues with interest in leadership and management. We have brought in younger colleagues into our DOTF and they have seized the moment and demonstrated their abilities and expertise. Some of our colleagues have similarly been seconded to institutional teams to lead and we in turn have had to identify additional talent to groom. A crisis of this dimension is a unique opportunity for nurturing future leadership talent [1].

\section{Conclusion}

The COVID-19 pandemic is a dynamic and fast-paced situation which mandates rapid response from all radiology leadership teams. We believe that by reconfiguring our management team into a tighter DOTF based on a military format has positioned us well to confront the many challenges to date within the context of our Singaporean experience. In managing the DOTF, discipline, flexibility, and teamwork are key principles, and these are built upon a strong foundation of focus on IPC, patient and staff safety, and staff well-being.
In formulating our response, the DOTF is also tightly aligned vertically and horizontally within our institution. While the military format may not be universally familiar, many of the principles we have embraced are widely applicable in effective crisis management. We are quietly confident that our experience to date in crisis leadership management will help us to navigate the complex issues that will arise with future surges in cases and in formulating strategies to manage exit from the present and future lockdowns.

Acknowledgments The authors acknowledge members of the disease outbreak task force (DOTF), Division of Radiological Sciences, Singapore General Hospital, for their valuable inputs and contributions, who include Dr. Png Meng Ai, Dr. P Chandra Mohan, Dr. Lionel Cheng, Dr. Lim Kheng Choon, Dr. Sivanathan Chandramohan, Dr. Lim Chee Yeong, Dr. Winnie Lam Wing Chuen, Dr. Charles Goh Xian-Yang, Dr. Apoorva Gogna, Agnes Wong Sau Kheng, Tan Chin Chong, Elias Woodrow Elias, Li Sheng, Danny Fooh, Kiew Yen San, Mindy Ang Choo Ping, Louis Teo Zhang Yi, John Tan Yeow Cheoong, Michelle Hia Xiao Hui, and Esther Wan Yee Shuang.

Funding information The authors state that this work has not received any funding.

\section{Compliance with ethical standards}

Guarantor The scientific guarantor of this publication is Dr. Kiang Hiong Tay.

Conflict of interest The authors of this manuscript declare no relationships with any companies whose products or services may be related to the subject matter of the article.

Statistics and biometry No complex statistical methods were necessary for this paper.

Informed consent Written informed consent was not required for this study because it is a special report on radiology leadership.

Ethical approval Institutional Review Board approval was not required because it is a special report on radiology leadership.

Methodology Not relevant.

\section{References}

1. Lexa FJ (2009) Leading in a crisis, part 1. J Am Coll Radiol 6:521-522

2. Gogna A, Tay KH, Tan BS (2014) Severe acute respiratory syndrome: 11 years later-a radiology perspective. AJR Am J Roentgenol 203:746-748

3. Revel MP, Parkar AP, Prosch H et al (2020) COVID-19 patients and the radiology department-advice from the European Society of Radiology (ESR) and the European Society of Thoracic Imaging (ESTI). Eur Radiol. https://doi.org/10.1016/j.ejro.2020.100237

4. Koh D (2018) 9 Occupational health aspects of emerging infections SARS outbreak affecting healthcare workers. Occup Environ Med 75: A14

5. Singapore Ministry of Health (MOH). $\mathrm{MOH}$ pandemic readiness and response plan for influenza and other acute respiratory diseases. 
Published 2004. Revised April 2014. Available via https://www. moh.gov.sg/docs/librariesprovider5/diseases-updates/interimpandemic-plan-public-ver- april-2014.pdf. Accessed 4 May 2020

6. Wong JEL, Leo YS, Tan CC (2020) COVID-19 in Singaporecurrent experience: critical global issues that require attention and action. JAMA 323:1243-1244

7. Cheng LTE, Chan LP, Tan BH et al (2020) Deja vu or jamais vu? How the severe acute respiratory syndrome experience influenced a Singapore radiology department's response to the coronavirus disease (COVID-19) epidemic. AJR Am J Roentgenol 214:12061210

8. Tsou IY, Liew CJ, Tan BP et al (2020) Planning and coordination of the radiological response to the coronavirus disease 2019 (COVID-19) pandemic: the Singapore experience. Clin Radiol 75:415-422

9. Tan BP, Lim KC, Goh YG et al (2020) Radiology preparedness in the ongoing battle against COVID-19: experience from large to small Singapore public hospitals. Radiology Cardiothoracic Imaging https://doi.org/10.1148/ryct.2020200140

10. Chen RC, Tan TT, Chan LP (2020) Adapting to a new normal? 5 key operational principles for a radiology service facing the COVID-19 pandemic. Eur Radiol. https://doi.org/10.1007/ s00330-020-06862-1

11. Mossa-Basha M, Meltzer CC, Kim DC, Tuite MJ, Kolli KP, Tan BS (2020) Radiology department preparedness for COVID-19: radiology scientific expert panel. Radiology. https://doi.org/10.1148/ radiol.2020200988

12. The Singapore Army. Available via https://www.mindef.gov.sg/ web/portal/army/about-the-army/organisation-structure. Accessed 4 July 2020

13. United States Army Field Manual 6.0. Commander and staff: organisation and operations. Available via https://armypubs.army.mil/ epubs/DR_pubs/DR_a/pdf/web/ARN14843_FM_6-0_Incl_C2 FINAL_WEB.pdf. Accessed 4 July 2020
14. The Editors of Encyclopaedia Britannica. General staff. Encyclopaedia Britannica, Inc. July 20, 1998. Available via https://www.britannica.com/topic/general-staff. Accessed 5 July 2020

15. VanVactor JD, Gill T (2010) Comparing military and civilian critical thinking and information processes in operational risk management: what are the lessons? J Bus Contin Emer Plan 4:97-112

16. Chen RC, Cheng LT, Lim JL et al (2020) Touch me not: physical distancing in radiology during COVID-19. J Am Coll Radiol 17: 739-742

17. Zhuang KD, Tan BS, Tan BH, Too CW, Tay KH (2020) Old threat, new enemy: is your interventional radiology service ready for the coronavirus disease 2019? Cardiovasc Intervent Radiol 43:665666

18. Too CW, Wen DW, Patel A et al (2020) Interventional radiology procedures for COVID-19 patients: how we do it. Cardiovasc Intervent Radiol 43:827-836

19. Gutzeit A, Li Q, Matoori S, Li B, Wang L (2020) What can European radiologists learn from the outbreak of COVID-19 in China? A discussion with a radiologist from Wuhan. Eur Radiol 30:3609-3611

20. Singapore Ministry of Health (MOH). Daily situation report. Available via https://www.moh.gov.sg/docs/librariesprovider5/ local-situation-report/situation-report\%2D\%2D-5-may-2020.pdf. Accessed 8 May 2020

21. Lexa FJ (2009) Leading in a crisis, part 2: succeeding in battle. J Am Coll Radiol 6:671-672

22. Kim H (2020) Outbreak of novel coronavirus (COVID-19): what is the role of radiologists? Eur Radiol 30:3266-3267

Publisher's note Springer Nature remains neutral with regard to jurisdictional claims in published maps and institutional affiliations. 